\title{
Development of a conceptually equivalent Chinese-language translation of the US Household Food Security Survey Module for Chinese immigrants to the USA
}

\author{
Christine ML Kwan ${ }^{1}$, Anna M Napoles ${ }^{2}$, Jeyling Chou ${ }^{1}$ and Hilary K Seligman ${ }^{1, *}$ \\ ${ }^{1}$ Center for Vulnerable Populations at San Francisco General Hospital; Division of General Internal Medicine, \\ University of California San Francisco, San Francisco, CA 94143, USA: ${ }^{2}$ Division of General Internal Medicine, \\ University of California San Francisco, San Francisco, CA, USA
}

Submitted 10 May 2013: Final revision received 19 December 2013: Accepted 31 January 2014: First published online 19 March 2014

\begin{abstract}
Objective: To develop a conceptually equivalent Chinese-language translation of the eighteen-item US Household Food Security Survey Module.

Design: In the current qualitative study, we (i) highlight methodological challenges which arise in developing survey instruments that will be used to make comparisons across language groups and (ii) describe the development of a Chinese-language translation of the US Household Food Security Survey Module, called the San Francisco Chinese Food Security Module.

Setting: Community sites in San Francisco, CA, USA.

Subjects: We conducted cognitive interviews with twenty-two community members recruited from community sites hosting food pantries and with five professionals recruited from clinical settings.

Results: Development of conceptually equivalent surveys can be difficult. We highlight challenges related to dialect, education, literacy (e.g. preferences for more or less formal phrasing), English words and phrases for which there is no Chinese language equivalent (e.g. 'balanced meals' and 'eat less than you felt you should') and response formats. We selected final translations to maximize: (i) consistency of the Chinese translation with the intent of the English version; (ii) clarity; and (iii) similarities in understanding across dialects and literacy levels. Conclusions: Survey translation is essential for conducting research in many communities. The challenges encountered illustrate how literal translations can affect the conceptual equivalence of survey items across languages. Cognitive interview methods should be routinely used for survey translation when such non-equivalence is suspected, such as in surveys addressing highly culturally bound behaviours such as diet and eating behaviours. Literally translated surveys lacking conceptual equivalence may magnify or obscure important health inequalities.
\end{abstract}

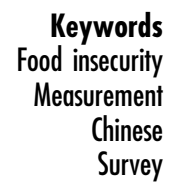

In $2011,85 \cdot 1 \%$ of US households were food secure (had access at all times to enough food for an active, healthy life for all household members) ${ }^{(1)}$. The other $14.9 \%$ of US households were food insecure, lacking adequate money or resources to reliably feed all members of their household for at least part of the year. National food security estimates are based on the Household Food Security Survey Module (HFSSM), originally developed by the US Food Security Measurement Project in response to the National Nutrition Monitoring and Related Research Act of 1990. The survey was developed by experts and refined after extensive cognitive testing, with subsequent years of research on scoring, stability over time, and robustness across population subgroups ${ }^{(2)}$. With minor revisions, the HFSSM has been administered approximately annually since 1995 as part of the US Census Bureau's Current Population Survey.

Food insecurity rates help to quantify the extent to which poverty in the USA affects food access and availability and the effectiveness of the nation's hunger safety net. The HFSSM has also enabled studies of the effects of food insecurity on family dynamics, dietary intake and health.

The US population consists of more than 38.5 million foreign-born individuals from 200 countries and territories $^{(3)}$. Food insecurity experts have called for increased research on how food insecurity affects population subgroups $^{(2)}$. Such research has been hampered by a lack of rigorous language translations of the HFSSM. The 
exception is the rigorous Spanish translation of the HFSSM by Harrison et $a l^{(4)}$. Their study highlighted important linguistic and conceptual difficulties in translating the survey, likely because normative dietary practices and eating behaviours are highly culturally bound.

The 'Chinese language' includes seven large language groups and hundreds of dialects. Grouped together, 'Chinese' is the third most common language in the USA, with nearly 2.5 million speakers ${ }^{(5)}$, and the second most common language spoken by Americans with limited English proficiency ${ }^{(6,7)}$. To understand food insecurity in Chinese Americans, our research group previously developed a word-for-word (literal) translation of the HFSSM. Anecdotal comments by interviewees suggested the literal translation might not be conceptually equivalent to the HFSSM. We were concerned that 'balanced diet' had a different meaning among English speakers $v$. Chinese speakers, with the Chinese interpretation potentially connoting traditional values of balance in hot and cold elements or balancing rice (a staple of the Chinese diet) with other foods. It was also unclear how Chinese speakers would interpret 'eat less than you felt you should', with the concept of how much one should eat being dependent on cultural norms and values. Finally, many Chinese-speaking households have personal experience with famine, particularly the 1958-1961 famine in China. These experiences may alter perceptions of what it means to be 'hungry'.

We therefore sought to develop a Chinese version of the HFSSM (called the San Francisco Chinese Food Security Module) that was conceptually equivalent to the English HFSSM and appropriate for administering to Chinese immigrants to the USA. We translated the existing survey, rather than creating de novo a Chinese-language food security survey, because the existing survey is broadly utilized and translation allows comparisons across language groups within the USA. The purpose of the present paper is to describe our translation methods and highlight methodological challenges which arise in developing survey instruments that will be used to make such comparisons.

\section{Materials and methods}

The HFSSM consists of eighteen items (Table 1). All respondents answer the first ten items, which reference household adults. Only households containing children answer the remaining eight items, which refer to children in the household. Two of the adult and one of the child items are skipped if the question preceding it is not answered affirmatively. (In self-administered versions of the survey, skip patterns are eliminated by collapsing items.) In addition, there is a six-item short form of the HFSSM which contains a subset of items in the full scale. This shortened scale is highly sensitive and specific for predicting food insecurity (using the eighteen-item version as the gold standard) ${ }^{(8)}$.

Items use three response formats: 'often true/sometimes true/never true'; 'yes/no'; and 'almost every month/ some months but not every month/only 1 or 2 months'. A unidimensional scale score is calculated by summing the number of affirmative responses ('often true', 'sometimes true', 'yes', 'almost every month' and 'some months but not every month'). A categorical food insecurity level is determined from this score ${ }^{(9)}$. For the eighteen-item scale, no affirmative responses denotes high food security; 1 or 2 affirmative responses, marginal food security; 3-7, low food security; and 8-18, very low food security.

Finally, a single-item screening question (the food sufficiency' item) is sometimes used to screen out participants who are unlikely to be food insecure ${ }^{(10)}$. This question is not part of the eighteen-item HFSSM and has its own response options: 'enough of the kinds of food we want', 'enough but not always the kinds of food we want', 'sometimes not enough to eat' and 'often not enough to eat'. Respondents reporting they have 'enough of the kinds of food we want' and have incomes more than twice the poverty threshold are assumed to be food secure $^{(11)}$. Our goal was to develop conceptually equivalent versions of the eighteen-, ten- and six-item versions of the HFSSM, in addition to the food sufficiency item, a total of nineteen items.

\section{Translation}

We focus on the two most prevalent Chinese dialects in the USA, Cantonese and Mandarin. Cantonese is common in Canton (Guangdong) province in southern China and Hong Kong, and has been historically dominant in Chinese communities in the USA. Mandarin is the official dialect of Mainland China and is increasingly common in the USA since the 1990s, corresponding to a wave of immigration from regions outside Canton. All Chinese dialects share a common written language using traditional or simplified Chinese characters.

We identified existing Chinese translations of the HFSSM through informal queries to experts and through searches for 'food insecurity' or 'hunger' and 'Chinese' on PubMed, www.google.com and www.scholar.google.com. We reviewed bibliographies of all manuscripts identified. We mailed letters requesting translations to the US Department of Agriculture, researchers who had published food insecurity studies conducted in Chinese-American populations ( $n$ 3), population-based survey administrators ( $n$ 2), and a nutrition and food insecurity researcher in Hong Kong ( $n$ 1). We received responses from all groups. Two groups had translated the six-item HFSSM into Chinese; both provided their translations, which were developed using a variation on a refereed single forward translation approach (without extensive cognitive testing) ${ }^{(12)}$. It was unclear in which Chinese dialect these written translations were initially developed. We therefore 
Table 1 Household Food Security Survey Module and food sufficiency item

\begin{tabular}{ll}
\hline Question & Response item \\
\hline $\begin{array}{ll}\text { Which of these statements best describes the food eaten in your } & \text { Survey(s) } \\
\text { household in the last } 12 \text { months? } & \begin{array}{l}\text { 2. Enough of the kinds of food we want } \\
\text { we want }\end{array} \\
& \begin{array}{l}\text { 3. Sometimes not enough to eat } \\
\text { item (screener) }\end{array}\end{array}$
\end{tabular}

Now l'm going to read you several statements that people have made about their food situation. For these statements, please tell me whether the statement was often true, sometimes true, or never true for (you/your household) in the last 12 months.

1. The first statement is: ' $(\mathrm{l} / \mathrm{We})$ worried whether (my/our) food would run out before (I/we) got money to buy more'. Was that often true, sometimes true, or never true for (you/your household) in the last 12 months?

2. 'The food that (I/we) bought just didn't last, and (I/we) didn't have money to get more'. Was that often, sometimes, or never true for (you/your household) in the last 12 months?
1. Often true
18-item
2. Sometimes true
10-item
3. Never true

1. Often true

18-item

2. Sometimes true

10-item

3. Never true

6-item

3. '(I/we) couldn't afford to eat balanced meals'. Was that often, sometimes, or never true for (you/your household) in the last 12 months?

1. Often true

2. Sometimes true

18-item

10-item

4. In the last 12 months, since last [name of current month], did (you/you or other adults in your household) ever cut the size of your meals or skip meals because there wasn't enough money

3. Never true

6-item

1. Yes

2. No

18-item

10-item for food?

5. If 'yes' to previous item: How often did this happen - almost every month, some months but not every month, or in only 1 or 2 months?

1. Almost every month

6-item

6. In the last 12 months, did you ever eat less than you felt you should because there wasn't enough money for food?

2. Some months but not every month

18-item

3. Only 1 or 2 months

10-item

1. Yes

6-item

2. No

18-item

10-item

6-item

7. In the last 12 months, were you ever hungry but didn't eat because there wasn't enough money for food?

1. Yes

18-item

2. No

10-item

6-item

8. In the last 12 months, did you lose weight because there wasn't enough money for food?

1. Yes 18-item

2. No 10-item

9. In the last 12 months, did (you/you or other adults in your

1. Yes 18-item household) ever not eat for a whole day because there wasn't enough money for food?

2. No

10-item

10. If 'yes' to previous item: How often did this happen - almost every month, some months but not every month, or in only 1 or 2 months?

1. Almost every month

3. Only 1 or 2 months

Now l'm going to read you several statements that people have made about the food situation of their children. For these statements, please tell me whether the statement was often true, sometimes true, or never true in the last 12 months for (your child/children living in the household who are under 18 years old).

11. ' (I/we) relied on only a few kinds of low-cost food to feed (my/our)

18-item (child/the children) because (I was/we were) running out of money to buy food'. Was that often, sometimes, or never true for (you/your household) in the last 12 months?

2. Sometimes true

3. Never true

12. '(I/We) couldn't feed (my/our) (child/the children) a balanced meal, because (I/we) couldn't afford that'. Was that often, sometimes, or never true for (you/your household) in the last 12 months?

1. Often true

18-item

2. Sometimes true

3. Never true

13. '(My/Our child was/The children were) not eating enough because (I/we) just couldn't afford enough food'. Was that often, sometimes, or never true for (you/your household) in the last 12 months?

1. Often true

18-item

2. Sometimes true

3. Never true

14. In the last 12 months, since [current month] of last year, did you ever cut the size of (your child's/any of the children's) meals

1. Yes

18-item because there wasn't enough money for food?

2. No 
Table 1 Continued

\begin{tabular}{|c|c|c|}
\hline Question & Response item & Survey(s) \\
\hline $\begin{array}{l}\text { 15. In the last } 12 \text { months, did ([child's name]/any of the children) ever } \\
\text { skip meals because there wasn't enough money for food? }\end{array}$ & $\begin{array}{l}\text { 1. Yes } \\
\text { 2. No }\end{array}$ & 18-item \\
\hline $\begin{array}{l}\text { 16. If 'yes' to previous item: How often did this happen - almost } \\
\text { every month, some months but not every month, or in only } 1 \text { or } \\
2 \text { months? }\end{array}$ & $\begin{array}{l}\text { 1. Almost every month } \\
\text { 2. Some months but not every month } \\
\text { 3. Only } 1 \text { or } 2 \text { months }\end{array}$ & 18-item \\
\hline $\begin{array}{l}\text { 17. In the last } 12 \text { months, (was your child/were the children) ever } \\
\text { hungry but you just couldn't afford more food? }\end{array}$ & $\begin{array}{l}\text { 1. Yes } \\
\text { 2. No }\end{array}$ & 18-item \\
\hline $\begin{array}{l}\text { 18. In the last } 12 \text { months, did (your child/any of the children) ever not } \\
\text { eat for a whole day because there wasn't enough money for food? }\end{array}$ & $\begin{array}{l}\text { 1. Yes } \\
\text { 2. No }\end{array}$ & 18-item \\
\hline
\end{tabular}

began our study with three versions (including our own) of the six-item HFSSM.

For each HFSSM item and response format, we compared existing translations. We discarded item translations that were obviously inconsistent with the intent of the English version ( $n$ 2). C.M.L.K. developed the initial translation for the food sufficiency item, four of the ten adult items (which had zero or one available translation) and the one child-referenced item which differed substantially from the adult-referenced items (item 11).

\section{Recruitment}

We conducted interviews with twenty-two Chinesespeaking immigrants to the USA. Because we were particularly concerned about how the items would be interpreted among low-income and food-insecure Chinese Americans, we recruited participants from food pantries hosted in a church, a multi-service agency and a senior housing building. We posted flyers and gave brief presentations to clients assembling at these same locations. Interested participants were asked to call a study researcher (C.M.L.K.), who administered a brief eligibility survey by telephone. Inclusion criteria included age $>18$ years and native fluency in Cantonese or Mandarin. Among interested callers, we purposively chose participants based on sociodemographic characteristics to ensure adequate representation by gender, age and dialect.

\section{Cognitive interviews}

All cognitive interviews were conducted by a bilingual and bicultural investigator (C.M.L.K.). C.M.L.K. is fluent in both Cantonese and Mandarin and received extensive training in food insecurity prior to conducting the study. Cognitive interviews lasted about an hour and were held during autumn 2012. Data consisted of extensive notes taken during interviews. Prior to the cognitive interview, a brief oral survey asked participants to self-report dialect preference, age, nativity, education, Chinese reading and writing proficiency, language preference for radio and television, and health status. Participants were compensated with a \$US 25 gift certificate to a local drug store. The study was conducted with written informed consent according to the guidelines laid down in the Declaration of Helsinki. The University of California San Francisco's Committee on Human Research approved all procedures.

The main technique employed in the face-to-face cognitive interviews was intensive verbal probing, which is recommended for exploring comprehension of survey items $^{(13)}$. This process was applied in three phases. First, all participants were read two translation versions of each question for all survey questions. After spontaneous responses were recorded, the interviewer immediately asked all participants probe questions: 'How clear were these questions?' and 'Do they mean the same thing to you?'. Depending on participant answers, the interviewer asked spontaneous follow-up probes, including why one item was clearer than the other, and how the items differed in meaning.

Second, in advance of the interviews, the research team identified certain words and phrases for additional probing because the concepts they conveyed were highly culturally bound, complex to translate, or presented difficulty during development of the Spanish version of the $\operatorname{HFSSM}^{(4)}$. Open-ended probes were developed in advance to explore the suspected problem with the translation of these specific phrases or words. The words and phrases identified for additional probing included: 'balanced meals', 'hungry', 'afford', 'cut the size of your meals', 'skip meals' and 'low-cost food'. To investigate comprehension of these phrases or words, we used several kinds of cognitive probes to elicit feedback: general probes (e.g. 'Tell me what you think about this translation'); comprehension/interpretation/connotation probes (e.g. "What does the term "skip meals" mean to you?' or 'How is the term "balanced diet" different from "healthy and varied diet"?'); and paraphrasing (e.g. 'Can you explain this translation to me in your own words?') ${ }^{(14,15)}$. These probes were asked of all participants until saturation was reached. Subsequent new probes were 
developed based on earlier cognitive interviews identifying new areas of concern or misunderstanding.

In the third and final phase of each interview, we asked interviewees to explain the meaning of each of the translated survey items in their own words with the probe, 'Please tell me in your own words what this item means to you'. Asking participants to paraphrase items is a commonly used probe technique ${ }^{(13)}$. If these explanations differed from the hypothesized intent of the English version, we asked interviewees for Chinese wording suggestions that better approximated the intent of the English version. One study researcher, an expert in food insecurity (H.K.S.), assisted with understanding intent of the English items. As there is little research into how English-proficient Americans understand the term 'balanced meal', we sought outside expertise regarding the intent and comprehension of this item (Mark Nord, PhD and John T Cook, PhD, MAEd, personal communication, 2012). We concluded that the translation of 'balanced meal' should communicate both the concept of eating healthy foods such as fruits and vegetables and that of eating a variety of food groups. Therefore, the English phrase corresponding to the Chinese phrase we sought was 'healthy and varied' rather than 'balanced'. Previous work translating the HFSSM into Spanish and Portuguese has also sought to match 'healthy and varied', rather than 'balanced', due to cultural differences in the way 'balanced' is understood ${ }^{(4,16,17)}$.

With one exception (item 11), HFSSM items referring to children are almost identical to items referring to adults. For these items, we used our near-final adult-referenced translations to create child-referenced translations. We then conducted one-on-one cognitive interviews with the complete eighteen-item survey (items referencing both household adults and children) and food sufficiency item and asked for additional feedback on clarity and conceptual meaning.

Finally, C.M.L.K. conducted cognitive interviews with five bilingual-bicultural (English and Chinese) professionals working in fields where they frequently discussed diet and nutrition with Chinese-speaking clients. These interviews included English and Chinese versions of the HFSSM. In addition to questions about clarity and meaning of the Chinese translation that paralleled those asked of community members, all of the professionals were also asked open-ended questions about the conceptual equivalence of the English and Chinese versions of each item. This was assessed for all participants with the probe, 'Can you tell me if you think these two versions mean the same or different things to you?'.

\section{Data analysis}

We conducted systematic item-level analyses. The interviewer collected notes for each item and probe response. The interviewer then used a systematic coding system to compare responses across interviewees. Specifically, for phase 1 in which participants were asked probes on the clarity and meaning of two translations of each item, codes that were applied were: (i) understood by participant $v$. did not understand $v$. not sure; and (ii) interpretation was inconsistent $v$. consistent with intended meaning. A note was also made if the participant volunteered alternative phrasing for the translation. For phase 2 in which specific a priori probes were developed for suspected problems with items, the responses to these probes were coded as interpretation was inconsistent $v$. consistent with intended meaning. Again, a note was also made documenting any suggestions by participants as to alternative phrasing for the translation. In phase 3 in which participants were asked to paraphrase items, the codes applied were: (i) understood by participant $v$. did not understand $v$. not sure; and (ii) interpretation was inconsistent $v$. consistent with intended meaning. Through in-person meetings, the research team then examined and arbitrated the coding until consensus was reached on the coding with respect to the comprehension of the items and evidence of the need to modify translations using suggested phrasing. Using an iterative process, these preliminary findings were reviewed with two investigators and discussed until consensus was reached on decisions to modify or leave items as is. In this way, responses from initial participants were used to replace inappropriate or awkward words and phrases with better ones; revisions were then presented to the next set of participants. For each item, we repeated the process until we reached data saturation, giving us a near-final version of the food sufficiency item and the ten items of the HFSSM referring to adults. Thus all participants were asked similar sets of questions, but for different survey items depending on the phase of the study. This is a commonly used technique for developing surveys in diverse populations ${ }^{(15)}$.

We selected final translations based on the following criteria: (i) the meaning of the Chinese translation was consistent with the intent of the item in English; (ii) the translation was clearly understood and not described as awkward in either dialect; and (iii) understanding of the translation was similar across dialects and literacy levels.

\section{Results}

We interviewed a diverse group of community members ( $n$ 22) and professionals ( $n$ 5). Community members were 34 to 80 years of age (Table 2 ). They were predominantly Cantonese speakers from Mainland China with an average duration in the USA of 18 years. Professional participants described themselves as a diabetes nurse, a clinical pharmacist, a nurse practitioner, a registered dietitian and a nutrition manager. They reported discussing diet in Chinese to an average of $5 \cdot 6$ clients/week (range 2-10).

Results of cognitive interviews are reported by the type of translation issue that surfaced in the interviews: (i) dialect; (ii) education and literacy; (iii) conceptual non-equivalence, or English words and phrases for which 
Table 2 Characteristics of community member and professional participants, San Francisco, CA, USA, autumn 2012

\begin{tabular}{|c|c|c|}
\hline & Community members ( $n$ 22) & Professionals $(n 5)$ \\
\hline \multicolumn{3}{|l|}{ Age (years) } \\
\hline Mean & 58 & 45 \\
\hline Range & $34-80$ & $36-57$ \\
\hline Female (\%) & 68 & 80 \\
\hline \multicolumn{3}{|l|}{ Country of birth (\%) } \\
\hline USA & 0 & 40 \\
\hline Mainland China & 82 & 0 \\
\hline Hong Kong & 14 & 60 \\
\hline Vietnam & 5 & 0 \\
\hline \multicolumn{3}{|l|}{ Preferred dialect (\%) } \\
\hline Cantonese only & 18 & 0 \\
\hline Mostly Cantonese, some Mandarin & 59 & 60 \\
\hline Mostly Mandarin, some Cantonese & 5 & 20 \\
\hline Cantonese and Mandarin equally & 18 & 20 \\
\hline \multicolumn{3}{|l|}{ Dialect of interview (\%) } \\
\hline Cantonese & 86 & 60 \\
\hline Mandarin & 14 & 40 \\
\hline \multicolumn{3}{|l|}{ Time residing in USA (years) } \\
\hline Mean & 18 & 28 \\
\hline Range & $3-40$ & $18-43$ \\
\hline \multicolumn{3}{|l|}{ Highest educational attainment (\%) } \\
\hline No school & 5 & 0 \\
\hline Elementary school & 5 & 0 \\
\hline Middle school & 14 & 0 \\
\hline High school (no degree) & 9 & 0 \\
\hline High-school degree/GED & 46 & 0 \\
\hline College or graduate school & 23 & 100 \\
\hline \multicolumn{3}{|l|}{ Place of highest education (\%) } \\
\hline USA & 9 & 100 \\
\hline Mainland China & 77 & 0 \\
\hline Hong Kong & 5 & 0 \\
\hline Vietnam & 5 & 0 \\
\hline \multicolumn{3}{|l|}{ Language use (\%) } \\
\hline Chinese only & 41 & 0 \\
\hline Mostly Chinese, some English & 46 & 0 \\
\hline Mostly English, some Chinese & 0 & 40 \\
\hline Chinese and English equally & 14 & 60 \\
\hline \multicolumn{3}{|l|}{ Radio/television exposure (\%) } \\
\hline Chinese only & 55 & 0 \\
\hline Chinese mostly & 32 & 0 \\
\hline English mostly & 0 & 60 \\
\hline Chinese and English equally & 14 & 40 \\
\hline Ability to read Chinese characters (\%) & 96 & 100 \\
\hline Ability to write Chinese characters (\%) & 96 & 100 \\
\hline \multicolumn{3}{|l|}{ General health (\%) } \\
\hline Excellent/very good & 9 & 60 \\
\hline Good & 36 & 0 \\
\hline Fair/poor & 55 & 40 \\
\hline
\end{tabular}

GED, General Educational Development.

there is no clear Chinese-language equivalent; and (iv) response formats. Unless otherwise noted, results refer to community member interviews.

\section{Dialect}

Cantonese and Mandarin speakers had different word preferences for some terms. Many interviewees spoke both dialects, so even for a given individual some preferences changed depending on whether the interviewee was 'thinking' in Cantonese or Mandarin. In general, Cantonese speakers preferred more casual words and phrases (for example, 買 for 'to buy') but found more formal words and phrases (購買 for 'to buy') acceptable. Mandarin speakers generally preferred formal words and phrases and sometimes found the casual ones inappropriate. In these situations, we chose the formal translation to maximize acceptability.

We encountered particular difficulty with dialect in the translation of 'ever hungry but didn't eat' (item 7). Cantonese speakers preferred one translation (挨餓) as more easily understood and less awkward, but Mandarin speakers found the phrase unfamiliar. We therefore opted for an alternative translation (感到飢餓) understood by speakers of both dialects.

\section{Education and literacy}

We examined variation in acceptability and clarity of translated terms by education and literacy level. For some terms, colloquial and formal translations were equally 
acceptable and clear at all levels of education and literacy (for example, 吃東西 or 進食 for 'to eat'). In these cases, we chose the more formal translation, as formal language is generally preferred in written text. When the formal phrasing was poorly understood by participants at lower education and literacy levels, we opted for colloquial phrasing (for example, 你有否 instead of 你有沒有 for 'did you').

\section{Conceptual non-equivalence (English words and phrases for which there is no clear Chinese-language equivalent)}

These individual words and phrases had no clear Chineselanguage equivalent: 'household', 'just', 'get more', 'meals', 'balanced' and 'eat less than you felt you should'.

The HFSSM asks responders to think about the food in their 'household'. When participants were asked what they called all the people who lived together and shared expenses, there was general consensus that this group was called 'family'. We therefore used the term 'family' in each of the HFSSM item translations.

A number of items included the word 'just' (e.g. 'the food we bought just didn't last'). This word also has no equivalent Chinese translation. We therefore omitted this word from the Chinese translation because we felt its use in the English version was superfluous.

We also had difficulty translating the term 'get more' (item 2). After testing multiple options, we used the term which translates to 'buy more' (買更多), which most closely approximates the hypothesized intent of the English version.

A literal translation of 'meals' (正餐) was generally interpreted as referring only to the most important meal of the day (either lunch or dinner, depending on the participant). There was a particularly strong response to the literal translation 'meals' in the context of 'balanced meals' (均衡的正餐), which participants interpreted as awkward, confusing and difficult to interpret. As an alternative, participants suggested a term that most closely approximates 'balanced diet' in English (均衡的飲食). This phrasing was clearly understood.

Because of considerable difficulty in translating 'balanced' (part of 'balanced meals', items 3 and 12) into Spanish, and persistent concerns that its interpretation varies considerably even among English participants, we queried multiple options for 'balanced' that corresponded to English variations previously tested: 'balanced diet' (均衡的飲食), 'healthy and varied diet' (健康和有多種類食物的飲食) and 'nutritious diet' (有營養的飲食). We initially asked participants to compare three translations and report differences in meaning and suggest other phrasing. Participants offered many options, but there was no consensus on which was most clear and conceptually similar to the English version. The 'healthy and varied diet' option was particularly difficult for participants. Some participants felt the words were redundant: 'a healthy diet presupposes a varied diet'. Others felt the words made little sense when put together: "When "healthy diet" and "varied diet" are put side by side, the phrase is difficult to understand. However, if you separate them then their meanings become clearer'.

Almost all participants recognized the phrase 'balanced diet' (均衡的飲食) in Chinese. Two descriptions of the meaning were common: to eat from different food groups ('to eat a bit of every group') or to eat foods other than those considered most palatable. Many (but not all) participants identified 'different food groups' as major food categories, including grains, protein, fruits and vegetables. Interviews with professionals confirmed community members' reports that the Chinese translation of 'balanced diet' was appropriate, acceptable and conceptually most similar to the English version.

We encountered difficulty translating 'eat less than you felt you should' (item 6). We initially considered three options from the previously translated surveys. The literal back-translations of these options were: (i) 'not eat to your fullness/not eat until you feel full...' (吃不飽); (ii) 'eat less than you felt you should...' (吃得比你覺得該吃的少); and (iii) 'not eat enough...' (進食不夠). We decided a priori to omit the third option because it clearly was not conceptually equivalent to the original item. We presented the other two versions to participants. Participants felt as if these two versions were equivalent in meaning, but that 'eat less than you felt you should' was 'too complicated in its wording' with very 'English' phrasing ('It sounds like an English expression. Chinese expressions don't read like this'). 'Not eat until you feel full' was 'simple and clear'. However, we preferred 'eat less than you felt you should' as participants described its meaning as closer to the intent of the English version. We therefore asked participants to improve on the wording of 'eat less than you felt you should' to be less cumbersome. Participants suggested removing the phrase 'you felt' (覺得). When offered 'not eat until you feel full' and 'eat less than you should', most participants still preferred the former but found the latter to be acceptable and understandable. We then asked participants to describe the meaning of 'eat less than you should'. Most descriptions were consistent with the English version, although this task was challenging for many participants.

All five professionals felt 'not eat until you feel full' and 'eat less than you should' conveyed different concepts, and that 'eat less than you should' was conceptually consistent with the intended meaning of the English item. Based on this feedback, our final Chinese translation is 'Did you ever eat less than you should...'.

\section{Response formats}

We found no difficulties with translation of the response stem 'How often did this happen?' and its response options. However, many participants found it difficult to understand the literal translation of the introduction to the other response option: 'Was that often true, sometimes true, or never true...?'. We therefore changed the response stem to the more easily understood: 'Did this situation often, sometimes, or never happen...?'. We changed the response 
options to 'often happened', 'sometimes happened' or 'never happened'. Participants preferred and understood this alternative translation.

\section{Final items}

We tested conceptual equivalence of the translated items by asking participants to give examples of situations qualifying for an affirmative response, for example situations in which you had to 'cut the size of your meals', 'skip meals' or '(rely) on only a few kinds of low-cost food'. All participants were able to describe appropriate examples.

Complete versions of the Chinese translation survey in multiple forms are available in the online supplementary material. Included are the food sufficiency item, six-item HFSSM, ten-item HFSSM and eighteen-item HFSSM formatted for verbal administration and self-administration.

\section{Discussion}

We used rigorous methodology to translate the US HFSSM into Chinese with the goal of developing a conceptually equivalent translation. The San Francisco Chinese Food Security Module prioritizes clear language (for items and response options), clarity across dialects and education levels, and phrases which approximate the intent of the English version. It varies substantially from a literal translation approach, emphasizing the need for such a process when surveys attempt to measure subjective and highly culturally bound constructs such as hunger, diet and eating behaviours. Translations without conceptual equivalence may introduce biases, such as suggesting that cultural differences are substantive when they are in fact semantic, obscuring or magnifying important health inequalities ${ }^{(18)}$. For example, we found in pilot studies using a literal translation of the HFSSM that the food insecurity rate among Chinese Americans was more than twice the food insecurity rate of other sub-populations. It is likely that this pilot finding reflects a high degree of measurement error due to conceptual non-equivalence across languages.

Little is known about how Chinese-American households cope with food insecurity. Our experience suggests that coping strategies may differ in Chinese communities. For example, interviewees mentioned two strategies not frequently observed in other sub-populations: drinking water to create a feeling of fullness and shopping just before closing time when the prices of foods at many stores in Chinatown are discounted.

In the Chinese culture (as in many cultures), food is not only a source of sustenance but also a critical component of social experience and general quality of life ${ }^{(19)}$. Food insecurity may thus have effects on physical and mental well-being that extend beyond dietary intake. Mixed methods or qualitative approaches would be helpful for exploring the cultural meaning and significance of food insecurity in Chinese-American households ${ }^{(20)}$.
The current work represents a much needed contribution towards the goal of allowing researchers to explore food insecurity and associated coping strategies in ChineseAmerican communities. Such exploration may allow targeted outreach to food-insecure Chinese-American households by food banks and government nutrition programmes. Establishing national benchmarks for tracking the number of food-insecure Chinese-American households may also illuminate the need for more resources in this community.

The Chinese-American population is highly diverse. Although 69\% of Chinese Americans are foreign-born, first-generation immigrants (many of whom do not speak, read or write English) ${ }^{(5)}$, many Chinese Americans have lived in the USA for generations and do not speak, read or write Chinese. Understanding food insecurity in the USA's 'Chinese community' will necessitate understanding how it is experienced in first-generation immigrant families as well as in highly acculturated families. Coping strategies are likely to vary considerably across this spectrum, similar to other immigrant groups.

Our study has several limitations. We conducted interviews in only the two most commonly spoken Chinese dialects in the USA. Because our intent was to design a survey that was understood by a diverse group of Chinese immigrants to the USA, we designed a single survey that was understood by primary speakers of multiple dialects. Dialect-specific translations would further maximize conceptual equivalence. This will be an important area of ongoing research. Our research suggested that the experiences of food insecurity among Chinese immigrants to the USA sometimes differed from the experiences of nonimmigrant populations and other immigrant populations to the USA. A new scale of food insecurity, rather than translation of an existing scale as we have done, may more fully capture the experiences of this population. Such de novo surveys may not facilitate comparison of food insecurity prevalence or coping strategies across large population groups. We recruited all interview participants from a single city (San Francisco). As participants were recruited from food pantries, the majority were food insecure. However, our professional sample allowed us the perspective of food-secure Chinese Americans. Further refinement of the scale should include the professional opinions of people who work in other fields, including social work, programme administration (e.g. food pantries) and advocacy. Until a validation study is completed, we do not know whether food insecure responses on the current survey correlate with changes in dietary intake or use of coping strategies, as they do in the English-speaking population. These represent important next steps.

\section{Conclusion}

In summary, we created a rigorously translated Chineselanguage version of the US Department of Agriculture's 
HFSSM called the San Francisco Chinese Food Security Module. This survey should catalyse efforts to understand the experience of food insecurity in Chinese-American communities. Our study draws attention to the importance of considering how issues related to dialect, literacy level, culturally bound or complex terms, and response formats can affect the conceptual equivalence of survey items across languages. It also calls for the routine use of cognitive interview methods in survey translation to illuminate when such non-equivalence may exist. Literally translated surveys which lack conceptual equivalence may magnify or obscure important inequalities.

\section{Acknowledgements}

Sources of funding: H.K.S. was supported by NIH/NCRR/OD UCSF-CTSI grant number KL2 RR024130. A.M.N. was supported by grant number P30-AG15272 from the Resource Centers for Minority Aging Research program of the National Institute on Aging. The contents of this paper are solely the responsibility of the authors and do not necessarily represent the official views of the National Institutes of Health. Conflicts of interest: None of the authors has any conflicts of interest. Ethics: The study was conducted according to the guidelines laid down in the Declaration of Helsinki and all procedures involving human subjects were approved by the University of California San Francisco's Committee on Human Research. Written informed consent was obtained from all participants. Authors' contributions: All authors designed the research; C.M.L.K. and H.K.S. conducted the research; H.K.S. and C.M.L.K. analysed the data and wrote the paper with assistance from A.M.N.; H.K.S. and C.M.L.K. accept primary responsibility for final content. All authors have read and approved the final manuscript. Acknowledgements: The authors thank each of the study participants for their assistance with the study, as well as St. John's Presbyterian Church, Donaldina Cameron House and the Chinatown Community Development Center (all based in San Francisco). They also thank Mark Nord, PhD (US Department of Agriculture) and John T. Cook, PhD, MAEd (Department of Pediatrics, Boston University School of Medicine) for their assistance.

\section{Supplementary material}

To view supplementary material for this article, please visit http://dx.doi.org/10.1017/S1368980014000160

\section{References}

1. Coleman-Jensen A, Nord M, Andrews M et al. (2012) Household Food Security in the United States in 2011. Economic Research Report no. ERR-141. Washington, DC: US Department of Agriculture, Economic Research Service; available at http://www.ers.usda.gov/publications/erreconomic-research-report/err141.aspx
2. Wunderlich GS \& Norwood JL (editors) (2006) Food Insecurity and Hunger in the United States: An Assessment of the Measure. Washington, DC: The National Academies Press.

3. Grieco EM \& Trevelyan EN (2010) Place of Birth of the Foreign-Born Population: 2009. American Community Survey Briefs; US Census Bureau, US Department of Commerce. https://www.census.gov/prod/2010pubs/acsbr09-15.pdf (accessed March 2014).

4. Harrison GG, Stormer A, Herman DR et al. (2003) Development of a Spanish-language version of the US household food security survey module. J Nutr 133, 1192-1197.

5. US Census Bureau (not dated) American Fact Finder, American Community Survey 2007-2011 Five Year Estimates. http://factfinder2.census.gov/faces/tableservices/jsf/pages/ productview.xhtml?src $=$ bkmk (accessed March 2014).

6. Pew Research Center (2013) The Rise of Asian Americans. Washington, DC: Pew Research Center; available at http:// www.pewsocialtrends.org/2012/06/19/the-rise-of-asianamericans/

7. DeNavas-Walt C, Proctor BD \& Smith JC (2011) Income, Poverty, and Health Insurance Coverage in the United States: 2010. Current Population Reports no. P60-239. Washington, DC: US Government Printing Office \& US Census Bureau; available at http://www.census.gov/prod/ 2011pubs/p60-239.pdf

8. Blumberg SJ, Bialostosky K, Hamilton WL et al. (1999) The effectiveness of a short form of the Household Food Security Scale. Am J Public Health 89, 1231-1234.

9. Bickel G, Nord M, Price C et al. (2000) Guide to Measuring Household Food Security. Alexandria, VA: US Department of Agriculture, Food and Nutrition Service; available at http://www.fns.usda.gov/fsec/FILES/FSGuide.pdf

10. Keenan DP, Olson C, Hersey JC et al. (2001) Measures of food insecurity/security. J Nutr Educ 33, Suppl. 1, S49-S58.

11. Turrell G (1996) Structural, material and economic influences on the food-purchasing choices of socioeconomic groups. Aust N Z J Public Health 20, 611-617.

12. Ponce NA, Lavarreda SA, Yen W et al. (2004) The California Health Interview Survey 2001: translation of a major survey for California's multiethnic population. Public Health Rep 119, 388-395.

13. Willis G (2005) Cognitive Interviewing: A Tool for Improving Questionnaire Design. Thousand Oaks, CA: Sage Publications, Inc.

14. Willis GB (1999) Cognitive Interviewing: A 'How To' Guide. Research Triangle Park, NC: Research Triangle Institute.

15. Napoles-Singer A, Santoyo-Olsson J, Brien H et al. (2006) Using cognitive interviews to develop surveys in diverse populations. Med Care 11, Suppl. 3, S21-S30.

16. Hromi-Fiedler A, Bermudez-Millan A, Segura-Perez S et al. (2009) Adaptation of the US food security survey module for low-income pregnant Latinas: qualitative phase. J Hunger Environ Nutr 4, 62-80.

17. Perez-Escamilla R, Segall-Correa AM, Kurdian Maranha L et al. (2004) An adapted version of the US Department of Agriculture Food Insecurity module is a valid tool for assessing household food insecurity in Campinas, Brazil. J Nutr 134, 1923-1928.

18. Sperber AD, Devellis RF \& Boehlecke B (1994) Crosscultural translation: methodology and validation. J Cross Cult Psychol 25, 501-524.

19. Chun KM \& Chesla CA (2004) Cultural issues in disease management for Chinese Americans with type 2 diabetes. Psychol Health 19, 767-785.

20. Kwan CM, Chun KM \& Chesla CA (2011) Cultural norms shaping research group interviews with Chinese American immigrants. Asian Am J Psychol 2, 115-127. 